\title{
Téoros
}

Revue de recherche en tourisme

\section{L'imaginaire géographique occidental du Grand Nord et la durabilité à l'épreuve du discours et des pratiques touristiques}

\section{Valérie Kohler}

Volume 31, numéro 1, 2012

URI : https://id.erudit.org/iderudit/1020721ar

DOI : https://doi.org/10.7202/1020721ar

Aller au sommaire du numéro

Éditeur(s)

Université du Québec à Montréal

ISSN

0712-8657 (imprimé)

1923-2705 (numérique)

Découvrir la revue

Citer cet article

Kohler, V. (2012). L’imaginaire géographique occidental du Grand Nord et la durabilité à l'épreuve du discours et des pratiques touristiques. Téoros, 31(1), 92-102. https://doi.org/10.7202/1020721ar

\section{Résumé de l'article}

Correspondant à de nouvelles attentes des touristes, l'écotourisme sert de label de qualité aux agences de voyages, notamment pour les destinations touristiques. L'Arctique n'y échappe pas. Les questions de durabilité, présentes dans le discours promotionnel de voyagistes, sont souvent mises en avant au nom d'un tourisme responsable. Cependant, les touristes occidentaux ne s'approprient pas toujours le discours des voyagistes, pas plus que les principes de développement durables préconisés par la gouvernance touristique à l'origine de ces offres. Le discours institutionnalisé pour une gestion touristique durable est mobilisé au niveau politique comme empirique en interrogeant la dimension écologique d'un tourisme arctique changeant. Qu'en est-il de l'intégration de ces principes de durabilité dans les pratiques de voyage, notamment dans le cas des croisières en milieux polaires, des activités dans les villages inuits ainsi que dans le discours des touristes ? À partir d'une étude de cas réalisée à bord d'un navire de croisière le long des côtes du Groenland, cet article s'intéresse aux décalages entre discours et pratiques sur la durabilité afin d'évaluer l'évolution de l'imaginaire géographique du Grand Nord par lequel le touriste construit son voyage et son rapport à l'altérité.
Ce document est protégé par la loi sur le droit d'auteur. L'utilisation des services d'Érudit (y compris la reproduction) est assujettie à sa politique d'utilisation que vous pouvez consulter en ligne.

https://apropos.erudit.org/fr/usagers/politique-dutilisation/ 


\title{
L'imaginaire géographique occidental du Grand Nord et la durabilité à l'épreuve du discours et des pratiques touristiques
}

\author{
Valérie KOHLER \\ Assistante doctorante \\ Département de géographie et environnement \\ Université de Genève \\ Valerie.kohler@unige.ch
}

\begin{abstract}
RÉSUMÉ: Correspondant à de nouvelles attentes des touristes, l'écotourisme sert de label de qualité aux agences de voyages, notamment pour les destinations touristiques. L'Arctique n'y échappe pas. Les questions de durabilité, présentes dans le discours promotionnel de voyagistes, sont souvent mises en avant au nom d'un tourisme responsable. Cependant, les touristes occidentaux ne s'approprient pas toujours le discours des voyagistes, pas plus que les principes de développement durables préconisés par la gouvernance touristique à l'origine de ces offres. Le discours institutionnalisé pour une gestion touristique durable est mobilisé au niveau politique comme empirique en interrogeant la dimension écologique d'un tourisme arctique changeant. Qu'en est-il de l'intégration de ces principes de durabilité dans les pratiques de voyage, notamment dans le cas des croisières en milieux polaires, des activités dans les villages inuits ainsi que dans le discours des touristes? À partir d'une étude de cas réalisée à bord d'un navire de croisière le long des côtes du Groenland, cet article s'intéresse aux décalages entre discours et pratiques sur la durabilité afin d'évaluer l'évolution de l'imaginaire géographique du Grand Nord par lequel le touriste construit son voyage et son rapport à l'altérité.
\end{abstract}

Mots-clés: Tourisme durable, stéréotypie, imaginaire géographique, exotisme, Arctique.

Les touristes portent un intérêt grandissant pour les destinations nouvelles ou inhabituelles comme le Grand Nord. Un séjour en Arctique nécessite pourtant une approche particulière vu la fragilité de son écosystème. Dans une perspective de conservation et de respect de l'environnement naturel et humain, des principes de développement durable ont été édictés pour les espaces de l'Arctique.

Dans ce cadre, des promoteurs de ces espaces proposent de l'écotourisme aux voyageurs à travers une appropriation de ces principes de durabilité. Par exemple, l'agence Wanderbird Expedition Cruises «offer[s] eco-tourism cruises that reflect our great reverence for the natural world and its creatures» (Miles et Miles, c2007-2012). D'autres agences telles que Ecosummer Expeditions n'hésitent pas à s'approprier le préfixe «éco-» pour vendre des voyages de nature ou d'aventure. Sur leurs sites Internet, la plupart des voyagistes mettent en avant, par le biais de diverses formes de critères écologiques à respecter, leur volonté de répondre à l'objectif environnemental du tourisme durable en Arctique. Sous le volet «À qui s'adressent nos voyages?», l'agence Grands Espaces (s.d.), fondée en 1998, souhaite «protéger nature et cultures locales» tout en faisant la promotion de ses circuits. Dans la section «développement durable» de son site, Grand Nord Grand Large (GNGL) (s.d.) déclare aussi être «très attentif à pratiquer des voyages respectueux des équilibres écologiques et humains» et avoir «à cœur de respecter nos hôtes, et leurs usages». GNGL, membre fondateur de l'Association pour un tourisme responsable (ATR), élabore une Charte éthique du voyageur dans l'Arctique dont les critères rejoindraient ceux d'un écotourisme actif.

À travers ces actions, les voyagistes montrent leur implication en tant que sociétés participant à la défense de l'environnement en marquant leur volonté de répondre aux objectifs du développement durable par des pratiques appropriées et suggérées à leurs voyageurs. Cependant, ce mode de gestion du voyage répond-il vraiment aux critères de l'écotourisme? Les pratiques touristiques les respectent-elles en regard de la réalité du terrain?

La problématique se situe aussi bien au niveau des voyagistes qu'à celui des voyageurs. D'une part, le tourisme arctique s'intensifiant (Grenier, 2009), l'engagement écologique de 
voyagistes spécialisés se trouve de plus en plus difficilement conciliable avec les objectifs de durabilité, notamment par l'augmentation de moyens de transport non écologiques lors d'activités touristiques. D'autre part, comment les touristes occidentaux reçoivent-ils ce discours des voyagistes incorporant la mise en ouvre de ces principes de développement durable à leurs offres? Jusqu'où les touristes les intègrent-ils dans leurs pratiques de voyage et comment en parlent-ils? Certains paradoxes entre discours et comportements de voyageurs visà-vis des populations de l'Arctique soulèvent ces questions.

Dès lors, dans quelle mesure les enjeux environnementaux, socioculturels et économiques de la durabilité modifient-ils le regard sur un Ailleurs exotique préconstruit tel que l'Arctique? L'imaginaire géographique joue ici un rôle clé dans les représentations qu'il véhicule, notamment dans l'historiographie de la conception qu'il entretient entre nature et culture.

Cette étude, réalisée à partir d'une enquête sur le terrain au Groenland, vise à relever les écarts entre le discours institutionnel de la durabilité dans le tourisme arctique et les pratiques touristiques effectives aussi bien de la part du voyagiste que du voyageur. Les objectifs du tourisme durable parviennent-ils finalement à impacter la réalité d'un marché arctique en pleine expansion?

\section{Méthodologie et contexte d'étude}

Durant l'été 2011, l'auteure a mené une étude qualitative de terrain fondée sur des entretiens et sur l'observation au Groenland de discours et pratiques de voyageurs des marchés suisse et français. Outre les méthodes d'observation participante et de discussions informelles, des entretiens semidirigés d'une heure et demie environ chacun ont été effectués en français auprès de 22 croisiéristes suisses francophones. Suivis régulièrement, les interviewés ont passé des entretiens à trois reprises correspondant aux trois phases de voyage de chaque interviewé, c'est-à-dire avant, pendant et après leur séjour commun de 15 jours passé dans l'Arctique groenlandais sur un bateau polaire de croisière. Dans une démarche réflexive d'investigation, l'auteure s'est référée au préalable à un guide d'entretien pour privilégier, au fil du travail de terrain, des entrevues semi-dirigées (sous forme de dialogues ouverts et spontanés tout en gardant une ligne imposée par des questions préparées à l'avance). Tous les entretiens ont été enregistrés à l'aide d'un appareil audio puis transcrits, tout comme les notes d'observation.

Cette étude s'inscrit dans le cadre d'une recherche de géographie culturelle sur les représentations occidentales du Grand Nord, notamment par la lecture touristique de voyageurs suisses en Arctique.

Un des axes de cette recherche concerne la question du développement durable et, plus précisément, son impact dans la construction de l'idée du Grand Nord chez les touristes, en regard de l'offre des voyagistes et de leur engagement pour un tourisme arctique durable.

Après un bref rappel historiographique des représentations occidentales du Grand Nord, cet article présente les principaux axes des directives de gouvernance pour un tourisme durable. En tant que forme de tourisme contemporain récent, le tourisme durable se définit par la prise en considération des principes du développement durable - en termes d'optimisation des ressources et de respect de l'environnement - appliqués aux activités touristiques. Le tourisme durable est étroitement lié, voire amalgamé, à son mode de gestion écotouristique. L'écotourisme se définit dans ses fondements comme étant «une forme de voyage responsable dans les espaces naturels qui contribue à la protection de l'environnement et au bien-être des populations locales» selon l'International Ecotourism Society (TIES, 1990). L'écotourisme s'inscrit dans un mode opératoire visant à rapporter des bénéfices tant au niveau de la conservation environnementale que sur le plan des retombées économiques positives du tourisme sur les communautés locales. Dans les offres des voyagistes, comment le tourisme arctique durable s'articulet-il alors avec cette pression de l'écotourisme, en regard d'une intensification du tourisme arctique?

Animés par un regard porté sur l'Autre et l'Ailleurs empreint d'exotisme, les discours des touristes permettent l'analyse de comportements concrets des voyageurs sur le terrain ainsi que la vérification du degré d'efficacité d'un tourisme arctique durable. Dans ses différentes déclinaisons, le tourisme durable fait émerger un caractère dit continu de l'imaginaire occidental du Grand Nord qu'il revêt et fait perdurer à travers des stéréotypes puissants. Certains aspects de cet imaginaire font en effet référence à des images stéréotypées récurrentes et indissociables de l'histoire de leurs représentations. Ainsi, l'écotourisme est une forme de tourisme à nuancer par l'origine contextuelle des représentations que s'en font ses usagers.

\section{Imaginaire géographique du Grand Nord : origine des représentations et stéréotypie}

Les représentations du Grand Nord vues par les Occidentaux sont imprégnées d'éléments mythiques et historiques constitutifs de l'imaginaire européen. La charpente sur laquelle se construisent ces représentations, fournit des indications sur le rapport à l'Autre, notamment en regard de la durabilité.

L'imaginaire des voyageurs suisses en Arctique se fonde principalement sur l'histoire occidentale de l'exploration européenne des XVIII et $\mathrm{XIX}^{\mathrm{e}}$ siècles dans ces contrées. Les brochures touristiques des voyagistes, comme Grand Nord Grand Large sur la page de couverture de son catalogue 2012, se réfèrent largement à ce symbole de l'imaginaire arctique. Les récits des explorateurs des siècles derniers et la restitution des représentations de leurs savoirs - notamment à travers les récits d'aventures qui s'en sont inspirés (McGhee, 2004) — ont largement contribué à construire les représentations de l'Arctique dans la vision occidentale. Cette construction s'est faite en particulier à l'aide du domaine géosémantique du Grand Nord «constitué d'une part d'un espace géographique, considéré comme cohérent, vaste et assez lointain, d'autre part d'un ensemble assez homogène de stéréotypes qui lui sont propres, qui participent de sa définition et, dans le cas de l'exotisme, de son attractivité» (Staszak, $2008: 20$ ).

Du mode spéculatif du "paradis perdu» comme âge d'or dans la pensée antique et médiévale, le mode de la réalité subjective s'inscrit dans le registre de la découverte et participe au renouvellement de la vision des espaces nordiques. Ainsi, l'imaginaire géographique européen catégorise d'abord le Grand Nord de manière prototypique, comme un espace froid 
et blanc. Tout en continuant à se focaliser sur le paysage et le climat, l'humain et le mode de vie inuit apparaissent ensuite comme des points d'intérêt anthropocentrés croissants dans l'imaginaire occidental. Traîneaux à chiens, chasseurs habillés en peau de bête, iglous se mêlent aux tempêtes de neige, déserts blancs et autres rappels paysagers comme les nomment les touristes sous les appellations de "vastes étendues» ou de "pureté du paysage» arctique. Ces stéréotypes de pensée (Schapira, 1999; Rémy, 1994) fixent «des croyances, des convictions, des idées reçues, des préjugés, voire des superstitions» (Schapira, 1999: 2) sur les zones géographiques appréhendées. L'ensemble de ces stéréotypes participe à cristalliser partiellement des représentations qui ressortent du discours touristique de la majorité des interviewés. Ainsi, avant leur voyage, les croisiéristes s'expriment sur les caractéristiques premières de l'Arctique :

Dans le Grand Nord... il fait froid et puis c'est blanc par-

tout et plat jusqu'à l'horizon. Une couverture de neige

plane comme un tapis blanc sans fin (Béatrice, 67 ans).

Alors moi, ça ne m'évoque pas grand-chose... si ce n'est

que je me dis, bien que voilà, il y a de la glace, il y a de

la neige, il fait froid... mais voilà, c'est tout (Gérard,

59 ans).

Je m'attendais à des paysages beaucoup plus blancs. Je m'attendais à quelque chose de tout blanc. Et puis, ce n'est pas tout blanc... (Alain, 63 ans).

Révélateur d'une manière de penser le monde, la portée symbolique de ces stéréotypes comme la blancheur fait du Grand Nord un «[o]bjet culturel construit par des siècles de discours qui ont fini par induire une simplification de ses éléments - blanc, loin, froid, en haut, inaccessible, pur et monstrueux, $[$ sic $]-[$,$] processus qui pourtant n'arrive jamais à en$ atténuer la pluralité, l'ambivalence et la complexité» (Chartier, 2008 : 5). En effet, les entretiens montrent que les voyageurs apportent certaines nuances à cette acception :

Alors le Grand Nord, pour moi, c'est les immenses étendues de... qui symbolisent pour moi la pureté. Je vois le Grand Nord comme quelque chose... Du blanc, le blanc, et toutes les nuances de blanc... Si tant est que l'on puisse dire que le blanc puisse avoir des couleurs, mais c'est vrai que, dans la glace, il y a toutes sortes de couleurs, qui vont du bleu au blanc pur... La mer, pleine qui charrie des glaçons, et le ciel bleu de préférence, mais... Ça me fait aussi penser au blizzard, au grand froid... (Georges, 72 ans).

C'est surtout l'inertie de ces symboles constitutifs des représentations du Grand Nord que l'on veut retenir ici, ainsi que leur perduration malgré des principes de développement durable dont les applications concrètes au niveau des pratiques observées semblent stagner. Les touristes n'évoquent prioritairement pas les atteintes faites à l'environnement lorsqu'ils parlent de l'Arctique. Les modifications de ce milieu devraient pourtant aussi façonner leur imaginaire, en réagencement permanent (Castoriadis, 1975; Debarbieux, 1995). Pourquoi la durabilité et ses applications concrètes peinent-elles à être intégrées par les touristes?

L'imaginaire du Grand Nord et les raisons qui motivent le voyage permettent d'apporter un élément de réponse, notamment par la déconstruction des stéréotypes directement liés aux pratiques culturelles des touristes sur les lieux concernés. L'imaginaire inspire et induit des comportements particuliers à travers le discours des voyagistes. En effet, la notion de désir et la tentation transgressive de la réalité tangible dans le monde des possibles font partie des clés de promotion du voyage. Les comportements se veulent donc également "détachés» d'une réalité réglementée et normative dont les espaces arctiques ne figureraient pas dans l'imaginaire voyageur.

Lors de discussions informelles et formelles dans une des questions d'entretien à propos des motivations de voyage en Arctique, les croisiéristes ne semblent pas faire des enjeux environnementaux (comme la lutte contre la pollution de l'air, des mers et des sols par des déchets parfois toxiques, entreposés en plein air, les dégradations et fuites chimiques que ceux-ci peuvent induire) une préoccupation majeure et encore moins une raison à leur déplacement dans l'Arctique. Ces problèmes sont pourtant dénoncés avant le départ en voyage par les futurs touristes, car ils sont connus et mentionnés à travers de nombreuses sources médiatiques ou des rapports gouvernementaux (MDAE et al., 2011 : 45). Les problèmes environnementaux constituent-il toujours un tabou pour les voyageurs du Grand Nord? L'analyse des entretiens montre une volonté des voyageurs, dans leurs motivations au séjour arctique, de mieux se départir d'une réalité imaginée par une connaissance approfondie donnée par l'expérience concrète du terrain. La croisière arctique contribue en outre à un désir profond de solitude partagée, mais aussi de distinction (Cohen, 1984) face à d'autres types de voyage, ouvrant ainsi la porte aux rapports de pouvoir. La nature, souvent perçue de manière figée dans le végétal et le minéral, est enfermée dans une image d'éternité et d'opposition à la condition moderne de l'humain d'aujourd'hui dans son ici :

Pour moi, c'est le rêve... Le Grand Nord, c'est quelque chose qui nous fait rêver, où je ne voudrais jamais vivre, là-bas, $[. .$.$] avec les traîneaux ou autres, parce que c'est$ trop froid [...] Je préfère garder le rêve de cette belle nature... une nature vraie... Pour moi, ça reste quelque chose de peut-être... inaccessible... ou d'extrême... (Régine, 66 ans).

Le touriste, complexe, est loin de n'être qu'un consommateur de voyage sans âme ou un «idiot du voyage» (Urbain, 1993). Par l'expérience inédite, ses désirs sont le reflet d'une vision barométrique du monde et s'inscrivent dans une combinaison de facteurs et de dimensions qui mènent à des paradoxes : selon les observations des touristes et de l'auteure durant le séjour, des déchets polluants sont visibles à même le sol dans divers sites et villages.

Le désir de nature intacte de l'imaginaire occidental pour ces espaces peut-il alors correspondre à un idéal de conservation sous-tendu par la durabilité? Avant d'analyser la prégnance du message de la durabilité sur les touristes, il convient de présenter les principes de gestion du tourisme.

\section{Principes de gouvernance et articulation aux offres du tourisme arctique}

Depuis une dizaine d'années, plusieurs modèles de gouvernance ont vu le jour : créée en 2003, l’organisation internationale Arctic Expedition Cruise Operators (AECO) a par 


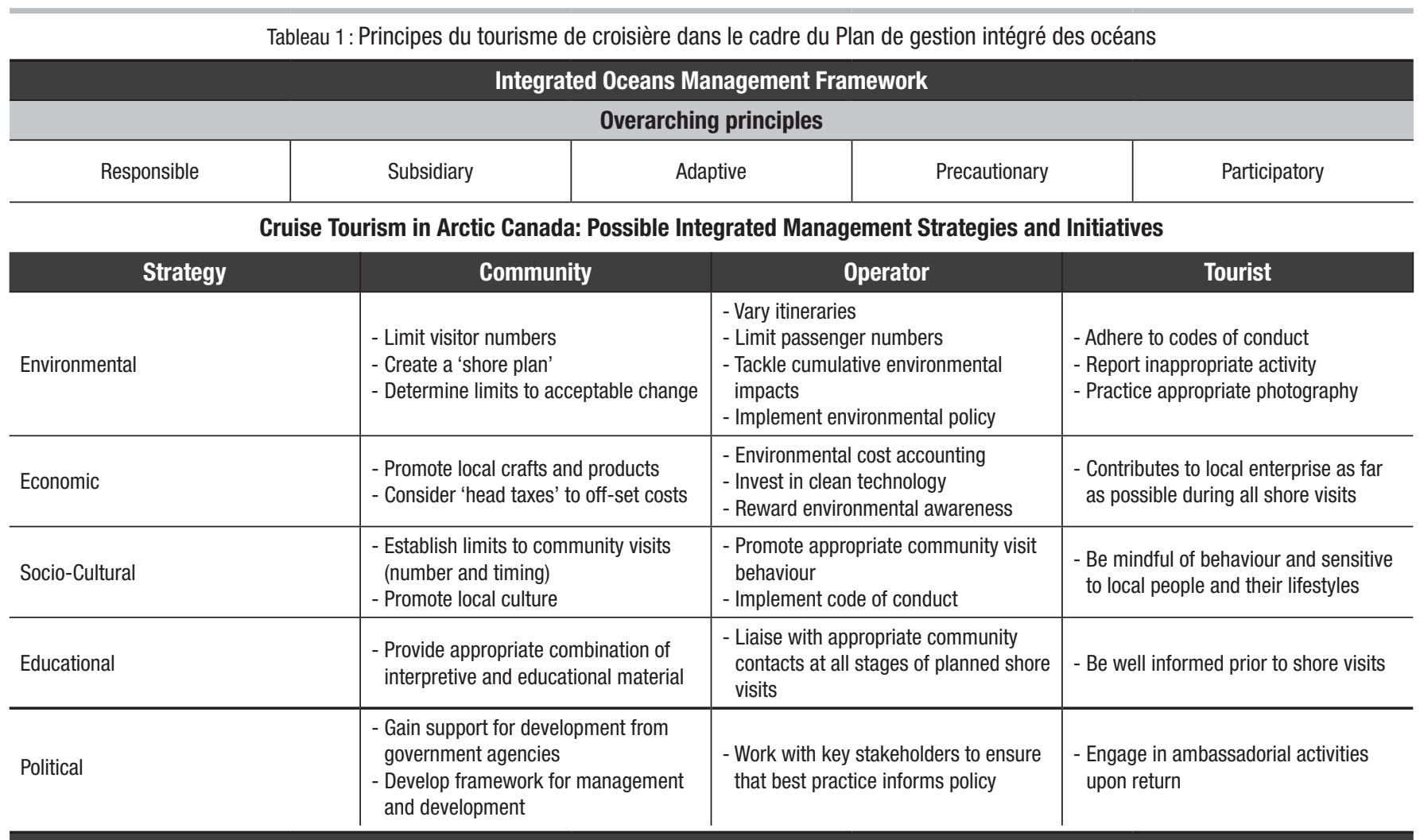

exemple permis de disposer d'un cadre légal formalisé et standardisé pour les bateaux d'expéditions scientifiques et touristiques dans les eaux arctiques (Dupré, 2009). Pour sa part, la Sustainable Arctic Tourism Association (SATA), fondée en 2005, soutient la mise en place de programmes de sensibilisation à plusieurs niveaux en vue de labelliser les produits durables de l'Arctique (Lasserre, 2010). Appliqués aux voyages promus par le tourisme durable, ces projets sont mis en place pour être avalisés par les communautés hôtes, mais aussi pour être appliqués dans leur intégralité à travers l'action des touristes.

L'International Association of Antarctica Tour Operators (IAATO) constitue également un repère important pour les voyagistes. En partenariat avec des associations faîtières, les actions initiées en faveur du respect des habitants du Grand Nord et de la protection de leur environnement font partie des messages principaux des sites des voyagistes. Créées par les agences de voyage, des fondations de soutien pour des projets locaux (aide humanitaire, soins médicaux, éducation, défense de la biodiversité) montrent l'importance de la visibilité de la durabilité touristique et de l'engagement des voyagistes. Outre les motifs concurrentiels et les intérêts commerciaux, ces derniers tentent de convaincre les clients potentiels du bien-fondé de leurs entreprises à l'heure où celles-ci sont à la recherche de nouvelles formes de légitimité (Bourdeau et Berthelot, 2008).
Le plan de gestion intégré des océans intitulé Integrated Oceans Management Framework (voir tableau 1), sur lequel se fonde l'Organisation Maritime Internationale, propose cinq principes de durabilité : responsable, subsidiaire, adaptatif, précautionneux et participatif. Ces principes sont des sources d'inspiration dans les stratégies et initiatives élaborées pour le tourisme de croisière dans l'Arctique.

Ce tableau reflète bien plus qu'une tentative d'élaboration de mesures pour endiguer les menaces qui pèsent sur les objectifs de la durabilité; cette proposition d'actions concrètes, au niveau des communautés, voyagistes et voyageurs, intégrées à un modèle en est la preuve. Couplée aux objectifs visant à satisfaire le touriste, elle préconise de limiter le nombre de passagers, de planifier les activités et de diversifier les circuits. Les visiteurs doivent quant à eux adhérer aux codes de conduite, dénoncer des activités considérées comme inappropriées qui auraient éventuellement eu lieu pendant le voyage et faire des photos jugées «adéquates» par les concepteurs de ce mode de gestion. Dans les faits, après analyse des entretiens et des observations de terrain, ces principes datant de 2002 semblent rester au stade d'une démonstration institutionnelle, sans anticipation quant aux résultats sur les comportements effectifs et le discours de la majorité des voyageurs interviewés.

Divers autres projets et programmes pour une pratique durable du tourisme polaire ont été lancés par le Conseil de l'Arctique. Ce forum intergouvernemental, fondé en 1996 
par la Déclaration d'Ottawa, envisage de promouvoir la coopération, la coordination, l'interaction et l'implication des communautés locales. Sans en détailler les principes, ses plans de développement durable, longtemps voués uniquement à l'environnement, intègrent tous une dimension tant économique que culturelle, inscrite dans la protection des cultures locales. La promotion d'une conception précise de la durabilité s'applique par des pratiques et conduites à adopter ou à éviter pour tous les acteurs concernés par les voyages dans l'Arctique. De surcroît, le message politique s'est donc massivement développé au sein de toutes ces actions de sensibilisation au développement durable.

\section{Tourisme arctique, tourisme de masse?}

Le développement du tourisme arctique représente un pan croissant d'un tourisme international en progression (OMT, 2011) dont la croisière océanique est l'illustration la plus marquante. En 2003, celle-ci regroupait déjà près de 10 millions de personnes (OMT, 2003). Le tourisme arctique englobe une typologie diversifiée de différentes approches qui se superposent ou se complètent, tel le tourisme d'aventure, culturel ou de nature incluant l'observation de la vie sauvage et la contemplation des paysages (Smith, 2009).

Les destinations septentrionales sont de plus en plus associées au concept organisationnel multidimensionnel d'écotourisme, dont les premières traces conceptuelles remontent aux années 1970 (Miller, 1978), et qui constituait déjà 40 à $60 \%$ du tourisme international dans les années 1990 (Fillion et al., 1992).

Dans ces diverses approches du tourisme arctique incluant une gestion écotouristique, le public des voyageurs ne correspond plus aux seuls marchés indépendants des randonneurs (backpackers). Aux formules du «tout compris» et «organisé en groupe» s'ajoute celle de voyages «à la carte». D'après les observations de terrain de l'auteure, l'Arctique devient une destination qui tend à se populariser parmi les offres à disposition, avec une diversification des classes sociales et des catégories d'âge des touristes concernés. Ainsi, ce ne sont plus les personnes de 60-70 ans qui se rendent dans l'Arctique, mais désormais de plus en plus de voyageurs dans la vie active, seuls, en couples ou en groupes d'amis. Il faut souligner que les activités liées au tourisme arctique s'inscrivent dans un contexte d'augmentation constante du tourisme mondial. Depuis les années 1980, le tourisme polaire connaît une réelle croissance. En 2007-2008, lors de l'Année polaire internationale (API), la diffusion de nombreux sujets documentaires, de conférences et d'autres événements, a contribué à la visibilité des régions polaires et donné une impulsion sans précédent pour ces destinations. Pourtant, les défis du développement touristique pour les communautés locales arctiques, en termes de retombées socio-économiques, sont encore à relever. De plus en plus important, le tourisme durable est «souvent vu comme une solution miracle capable de concilier le développement économique, la protection environnementale et le bien-être des communautés» (Tardif, 2003 : 1). La médiatisation des possibilités marchandes de traverser les Passages du Nord-Ouest (Canada) et du Nord-Est (Russie) a une influence considérable dans la réduction des distances et les itinéraires à venir, notamment pour un tourisme dont le mode de transport privilégié reste la navigation.
La demande globale de voyages maritimes s'est donc accentuée ces 30 dernières années : le tourisme de croisière a plus que doublé en 10 ans, entre 1995 et 2005, pour atteindre l'estimation de 16 millions de passagers en 2011 (FCCA, 2011; OMT, 2003). Le Costa Luminosa et le Costa Deliziosa, dont la capacité peut atteindre plus de 2200 passagers, se sont respectivement rendus à Ilulissat dans l'Ouest groenlandais aux mois d'août 2010 et 2011. Le tourisme arctique se diversifie dans ses offres pour satisfaire la demande constante de repousser les limites dans l'atteinte de destinations encore vierges de tout passage touristique. Anciennement considéré comme extrême, l'archipel norvégien du Svalbard, à $78^{\circ}$ de latitude nord, n'est plus la seule destination la plus prisée dans ce type de voyage. Sa capitale, Longyearbyen, accueille plusieurs de ces paquebots, dont le Costa Pacifica avec ses 3780 passagers à son bord, venu trois fois durant la saison estivale de 2012, selon la Cruise Ship List (2012) du port de Longyearbyen.

L'exemple du Groenland est emblématique du point de vue du développement touristique. Statistics Greenland (1989) dénombre une augmentation constante depuis 2002, avec plus de 35000 touristes internationaux en 2005. Dès lors, les nuitées en hôtellerie classique sont aussi en progression, tout comme l'effectif des bateaux de croisière, passant de 15654 passagers en 2004 à 30271 en 2010 (MDAE et al., 2011 : 18). En 2011, le nombre de visiteurs par an est estimé aux deux tiers de la population locale d'environ 56000 résidents. Principale ville touristique, Ilulissat a vu 42 passages de bateaux durant l'été 2011 avec un accostage tous les 2,7 jours en moyenne, sur un total de 282 passages pour le Groenland à la même période. Ces estimations sont fondées sur le relevé des passages de bateaux de croisière par ville et par village groenlandais, d'après la liste publique Anløbsliste (2011).

La comparaison entre le nombre d'habitants des lieux visités et les effectifs de voyageurs, à l'échelle locale aussi bien que régionale et nationale, amène à noter l'importance du concept d'échelle dans le cadre des actions pour la durabilité. Certains auteurs n'en tiennent pas compte en considérant que l'action durable a un impact indépendamment d'autres niveaux d'observation (Weaver : 2002). Cependant, une prise en considération de l'échelle permettrait de montrer l'ampleur du fait suivant : le tourisme arctique par voie maritime est le secteur touristique dont la croissance est la plus forte (Lück et al., $2010: 2$ ) non seulement en nombre de passagers par an, mais aussi par la taille même des bateaux, véritables villes flottantes se rendant dans les eaux libres de glace du Haut-Arctique.

Cette hausse de fréquentation du Grand Nord s'explique entre autres par des éléments tels que la popularisation touristique, l'adaptation des pays hôtes, les influences médiatiques et politiques, ou encore l'accessibilité facilitée.

Ce constat permet-il de classer le tourisme arctique au rang d'un tourisme de masse? L'Arctique reste un espace privilégié peu visité, comparé aux nombreuses destinations standardisées. Dans ce cadre spécifique, les voyagistes utilisent le label «éco-» touristique au service d'une promotion du tourisme durable, tel que Ecosummer Expeditions (1976) ou encore World Expeditions (s. d.) qui exprime dans son volet «Community Project»: «World Expeditions has a vision - to make the world a better place for people in need. Voluntourism; 
Eco Tourism - no matter what you call it, we would like to invite you to share in this vision".

Contrairement au tourisme de masse, le tourisme arctique a besoin du levier de l'écotourisme au service d'un marketing plus efficace pour garantir la vente de voyages, en général onéreux. Malgré sa forte croissance, ces facteurs permettent donc d'écarter la qualification de masse au tourisme arctique, pour autant qu'il défende l'application des principes du développement durable.

\section{Facettes multiples de l'écotourisme}

L'accueil d'un nombre plus élevé de touristes au fil du temps ferait en effet chanceler le respect des principes de la durabilité soutenus par l'écotourisme, selon la définition de l'International Ecotourism Society (TIES, 1990). Bien qu'il ne fasse pas l'objet d'une charte officielle ou d'une marque déposée, ce concept fait office de label pour le développement du tourisme arctique. Il est alors librement utilisé par les voyagistes spécialisés pour offrir des séjours correspondant aux critères des programmes et des directives de la gouvernance environnementale. Cependant, peut-on vraiment parler d'écotourisme en ce qui concerne le tourisme arctique, notamment de croisières? Celles-ci se fondent sur une approche de voyage principalement tournée vers le spectacle de la nature et les émotions qu'elle suscite. Les préfixes «éco-» ou «bio-» résonnent comme les promoteurs d'un nouveau marché dans le renouvellement de ce type de tourisme. Leurs protagonistes font partie des " ecotourists on tours» d'après une typologie de Kusler (1991) fondée sur les motivations et les expériences touristiques.

Suite à la multiplicité et à l'intensification de ces offres de voyages dans leur mode, leur type et leurs destinations, le tourisme polaire est à la recherche de concordance face aux nouveaux défis d'une politique environnementale plus normative. Le terme d'écotourisme est assez récent : l'expérience du tourisme d'aventure prédomine avant que les préoccupations écologiques et socio-économiques ne se renforcent de manière concrète sur l'ensemble des offres touristiques.

Le tourisme arctique peut intégrer un type d'écotourisme «doux» (Fennell, 2008) et voit ses principes de durabilité remis en question par les impacts directs des bateaux de croisières naviguant dans les mers de hautes latitudes. Bien que des réglementations en zones polaires existent et que certains navires, comme Le Boréal, prennent des précautions comme l'entreposage de ses déchets à bord, il n'en reste pas moins que les bateaux utilisent tous des ressources d'énergie fossile plus ou moins allégée pour leur fonctionnement. Outre le type de navigation et d'activités lors de leur parcours, le nombre de ces bâtiments présents augmente. La pollution et les émissions ainsi que la dégradation biologique des écosystèmes environnants croissent proportionnellement. Les risques de collisions avec les icebergs les exposent aux accidents (Grenier, 2008), ajoutant une difficulté supplémentaire à la crédibilité des codes de conduite à respecter en matière d'écotourisme. Par leur caractère exemplaire, ces éléments incitent donc à relativiser l'écotouristicité du milieu arctique. Celle-ci présuppose la mise en cohérence entre une philosophie du voyage désirée par le touriste et les dispositions prises pour accéder à sa destination. En d'autres termes, la fin ne devrait pas justifier les moyens.
Amorcé à la fin des années 1980 par le rapport Brundtland de 1987, l'engagement écologique de la part des voyagistes est progressivement apparu depuis la nécessité d'un développement durable à l'échelle mondiale "qui répond aux besoins des générations du présent sans compromettre la capacité des générations futures à répondre aux leurs» (United Nations, 1987). Cette prise de conscience du caractère fini de la Terre et de l'épuisement irrémédiable de ses ressources a engendré une nouvelle conception du bien public et du devenir social, économique et environnemental de la planète. Publicités télévisuelles, chartes, logos, codes de conduites, actions bénévoles et autres campagnes pro-environnementales accompagnent désormais les sites Internet des voyagistes, dont les médias se font largement le relais du message écotouristique et dont les controverses ont été éclairées par Gössling (2000). Vecteur principal de communication, la culture du Web apporte aujourd'hui un outil de compréhension important des transformations qui s'opèrent dans les relations entre l'internaute et l'objet touristique qu'il convoite. L'Internet permet un suivi des aspects directement liés à la destination de voyage, notamment dans les représentations touristiques qu'il rend visibles par des sites tels que TripAdvisor.

Si l'ensemble des touristes perçoit le Grand Nord comme «intouché», «unique», "grandiose» ou «spectaculaire», aucun des participants de l'échantillon ne mentionne en revanche les changements vers une durabilité environnementale, économique ou sociale. La découverte d'éléments imprévus sur le terrain, tels que les désagréments causés par les odeurs de poissons séchés ou les horaires non respectés dans la programmation d'activités locales, est immédiatement relevée par certains des interviewés comme des imperfections au voyage "clé en main».

La tendance au «tourisme-système», auquel le voyageur est habitué à se référer, peut conduire à disjoindre actes et discours en gelant le processus d'application de la durabilité; le touriste est incité à reproduire un "modèle du voyager» connu, déjà testé et éprouvé, sans pour autant en approuver le fond.

Les représentants de la gouvernance mondiale émettent des recommandations afin de mettre en œuvre des modes de pilotage pour répondre aux problèmes environnementaux. Bien que l'offre touristique prenne en compte ces paramètres, les voyageurs semblent les reléguer au second plan au profit d'un imaginaire stéréotypé.

\section{Tourisme de nature et de culture ou I'illusion d'un tourisme arctique durable}

Un décalage est observable entre les recommandations des voyagistes découlant des directives pour une gouvernance durable et leur écho auprès des touristes. Lors de l'étude de terrain de l'auteure sur le navire, le responsable de la croisière effectuée a introduit le programme du voyage et de ses lieux sous l'angle du «respect inuit» avec le message implicite d'égalité culturelle, de telle sorte que les touristes adoptent un comportement approprié, car «les Inuit[s] saluent les gens, même les touristes...» (Kohler, 2011 : note d'observation). Pourtant, l'application de ces principes de gestion intégrés au comportement touristique de visite est parfois bien vite oubliée. Observer la nature est déjà pris comme une bonne 




ILLUSTRATION 1 : Démonstration de maquillage de masque facial et de danse au tambour par une artiste groenlandaise, llulissat (photo : Valérie Kohler).

pratique par certains touristes, qui ne nomment jamais le tourisme durable, pas plus que l'étiquette d'écotouristes à laquelle ils ne s'identifient pas, comme constaté dans les discussions informelles de l'étude. Ils confondent ainsi volontiers l'écotourisme et le tourisme de nature propre à leur séjour. Voir, c'est faire sans faire.

Sur le terrain, l'information sur le comportement à adopter durant les visites vis-à-vis des communautés locales s'avère même nécessaire : «ce sont des personnes», comme devait le préciser l'un des responsables francophones d'une agence de voyage juste avant la visite d'un village groenlandais à l'extrême nord-ouest du pays. Les touristes ne suivent pas toujours ces conseils; s'ils ne dégradent pas l'environnement visité par le dépôt sauvage de déchets, ils n'hésitent cependant pas à photographier les Groenlandais de manière excessive en provoquant des signes clairs de désapprobation de la part de ces derniers. Relativement bien informées dans la mise en œuvre et le contrôle de ces principes, les agences sont alors directement confrontées aux impacts négatifs d'une industrie touristique croissante dans ces régions. Sous l'effet de leur imaginaire, les touristes contournent l'application d'un code de conduite en phase avec les objectifs de durabilité. Les participants adoptent la position de néoexplorateurs rapportant un trophée modernisé par le biais de la photographie. Ainsi, les objectifs de «capture» de l'Autre sont assouvis comme objet central de cette mise en scène touristique. Les populations locales sont souvent hésitantes dans le mode de comportements à adopter — en regard notamment des enjeux commerciaux que ceux-ci entraînent — et les Inuits n'acceptent pas toujours de «jouer le jeu » au point de se livrer à des expressions culturelles forcées pour satisfaire l'attente des étrangers de passage. C'est sous la forme d'un partage des retombées économiques touristiques que le voyageur devrait s'engager pour la culture de l'Autre, selon les dires d'une Inuk impliquée dans la vie touristique d'Ilulissat.

La réponse à des comportements touchant à l'intégrité des personnes peut être vive, immédiate et réflexive. Par exemple, la prise en photo d'une institutrice inuite entourée de ses élèves âgés de 5 à 10 ans par un groupe d'une vingtaine de touristes, et ce, à son insu, a provoqué une réaction inattendue pour ces derniers : ce que cette femme a considéré comme un abus, elle l'a à son tour infligé aux touristes en question. Ces derniers, surpris d'être photographiés, sont rentrés interpellés de cette forme d'expression à leur égard. Le mythe du «bon sauvage» n'est pas prêt d'être enterré; il perdure dans l'imaginaire où l'Autre est vu comme dernier témoin préservé dans et par un milieu naturel tout aussi intact. L'expérience a déstabilisé temporairement les repères du touriste. Celui-ci se met ainsi en scène, quitte à outrepasser ses droits qu'il attribue à l'Ailleurs au nom d'un exotisme revendiqué dans la réalisation de son voyage. L'Autre en rébellion constitue une transgression dans l'imaginaire occidental du Grand Nord, symbolisé entre autres par la femme exotique, gentille, patiente, docile, voire servile qu'elle représente (Staszak, 2008). Cette prise de conscience du traitement infligé à la villageoise tel un objet touristique plutôt qu'un être humain respectable a momentanément dévié les touristes de l'emprise de leur imaginaire.

Malgré les discours sur les codes de conduite et les mises en garde de la part des agences touristiques ainsi que de leurs représentants sur le terrain, le touriste agit d'abord dans la conception qu'il se fait de son voyage, dans une mise en scène qu'il s'imagine bien avant son départ. En se fondant sur une vision propre aux empires coloniaux européens dans leur ensemble, la dominance de l'esprit colonial des XIX et $\mathrm{XX}^{\mathrm{e}}$ siècles a largement participé à propager les stéréotypes qui l'accompagnent dans le monde occidental et dans les pays dits non coloniaux, comme la Suisse ou le Luxembourg. Les formes d'exotisation sont nombreuses. Entretenues par les stéréotypes, elles font vaciller les principes fondamentaux de la durabilité sociale et culturelle tels que les conçoivent les modèles actuels de gouvernance. Plusieurs activités touristiques profitent à la stéréotypie que celles-ci sous-tendent, provoquant des ambiguïtés entre discours et principes de développement durable, regard porté sur l'Autre et apports économiques aux communautés locales.

Ces écarts se retrouvent sous forme de lieux et d'expressions culturelles proches d'une survalorisation de l'objet touristique. Seuls des dispositifs bien précis tels que musées, magasins de souvenirs et événements locaux — improvisés ou non - sont censés participer officiellement aux bénéfices socio-économiques des localités arctiques, promulgués par les recommandations d'un tourisme responsable. En revanche, dans les faits, un danger de marchandisation culturelle existe lors de ces visites touristiques, notamment dans les modifications du rapport qu'entretiennent les Inuits avec leur territoire; ces populations sont en effet encore en phase de 
mutation profonde (Collignon, 2005), et les impacts sociaux et familiaux sont d'autant plus préoccupants que la tendance du tourisme arctique est à la hausse. Dans ces transformations sociétales, l'exemple de la théâtralisation de l'objet culturel au service du tourisme montre non seulement un risque de dérive, mais peut aussi, à l'inverse, favoriser l'émergence d'actions alternatives.

La "danse masquée» (masked dance) constitue un exemple d'expression artistique de la culture du Groenland - dont la côte ouest est d'abord visitée pour ses icebergs et le fjord d'Ilulissat classé à l'UNESCO en 2004. La signification culturelle de cette danse glisse peu à peu vers un art scénique récupéré par les acteurs économiques au titre d'activité touristique. Une comédienne professionnelle inuite monte ainsi des spectacles pendant l'été, en passant par toutes les étapes de démonstrations et de leurs récits associés, telle la préparation à la danse par le maquillage (voir illustration 1).

Outre le mélange culturel et artistique de la danse, la restitution orale des mythes et des histoires groenlandaises apportent aux touristes l'impression de participer à une meilleure compréhension de l'Autre. En effet, la représentante directe de la culture inuite correspond à l'image stéréotypée et attendue des touristes au sens où l'entend Wang (1999 : 355) dans sa référence à l'authenticité. La relation au corps exotique est aussi un point important à mentionner car «le spectacle est non seulement une représentation mais aussi une présentation de son corps» (Staszak, 2008 : 18). Associée à l'étrangeté du pays lointain, la démonstration est teintée d'érotisme que la danseuse suggère par l'exotisme qu'elle suscite aux yeux des touristes. Cette forme d'exotisation de l'Ailleurs, avec l'expression corporelle et son érotisation à travers les spécificités du désirable et de l'agréable, fait partie du fantasme de l'authenticité recherchée dans l'Ailleurs. Il se voit aussi à travers les objets souvenirs ou les arts picturaux danois traitant de l'Arctique. Ainsi, l'image de la déesse Sedna, considérée comme une des divinités les plus connues par les Inuits et représentée par une sirène, a été reprise par l'artiste danois Gitz-Johansen (1897-1977) dans une lithographie intitulée Ímap Ukuâ. Le dessin retravaillé par son auteur sous la forme d'une femme intégralement nue au visage peint fut reproduit en masse sur des $t$-shirts aux couleurs vives, destinés à la vente touristique (voir illustration 2). Cet artiste a peint à plusieurs reprises des femmes groenlandaises dénudées, telle que l'œuvre intitulée Thule bopladsens Madonna (Madonne de la colonie de Thulé) que l'on retrouve sous forme d'affiche à vendre aux visiteurs du Musée d'art d'Ilulissat, ou encore au magasin de souvenirs du village d'Upernavik.

Ajout de l'exotisme et fabricant d'altérité pour les anciens Empires, l'érotisme est par exemple animé de stéréotypes attendus par les touristes. Issu du passé colonial, le regard porté sur la gente féminine de l'ailleurs persiste parfois au-delà des discours sur la durabilité. En effet, les principes du développement durable induisant le respect des populations inuites, de leur mode de vie et de leur culture mènent à un rapport de force entre la stéréotypie puissante de l'Arctique et les processus de désexotisation de celui-ci.

La stéréotypie occidentale du Grand Nord concerne aussi l'habitat : certains touristes continuent de penser que

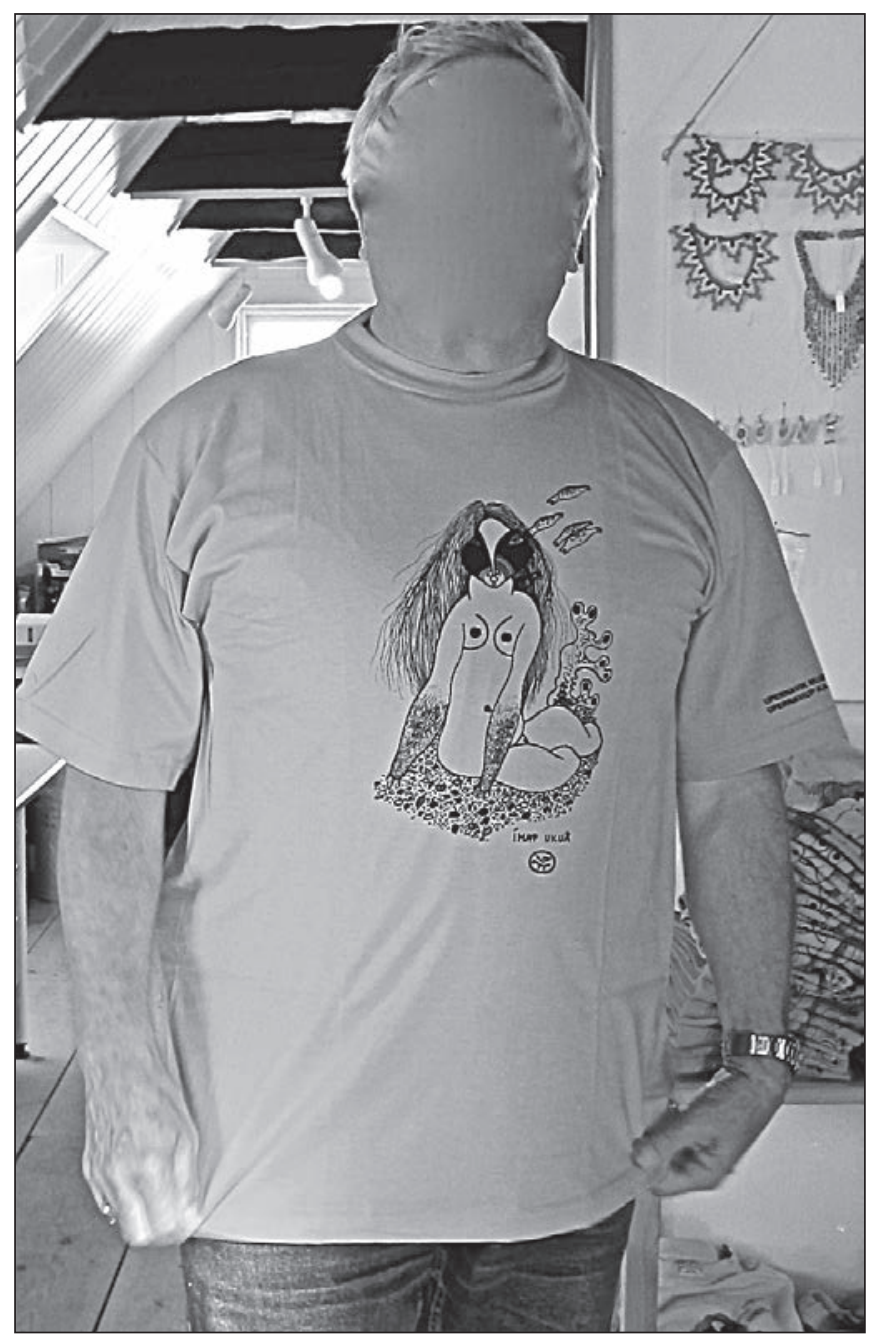

ILLUSTRATION 2 : Touriste portant un t-shirt sur lequel figure la reproduction d'une lithographie de l'artiste danois Aage Gitz-Johansen (1897-1977), Upernavik (photo : Valérie Kohler).

les Inuits habitent quotidiennement dans des maisons en forme d'iglou, même au Groenland où il n'a jamais été une forme de construction typique. Certains hôtels entretiennent pourtant cette image pour offrir des chambres d'hôtel en forme d'iglou. Les magasins de souvenirs vendent également objets et bijoux d'artisanat reproduisant l'habitat mythique (voir illustration 3). Ainsi, la stéréotypie dans l'art inuit laisse apparaître la potentialité de ces objets (Graburn, 2004) et peut révéler une volonté d'affirmation économique, culturelle, politique, en revalorisant des pratiques culturelles locales par le tourisme. La volonté de montrer une image positive et moderne émane aussi de la jeune génération des Groenlandais aujourd'hui.

En plus des questions de genre et des éléments relatifs à la danse, l'habitat ou les objets, la démonstration du tambour traditionnel est un autre exemple où la durabilité semble n'être qu'un caractère exacerbé de la stéréotypie. Selon les explications d'un Inuk sur place, le tambour groenlandais - fait en 


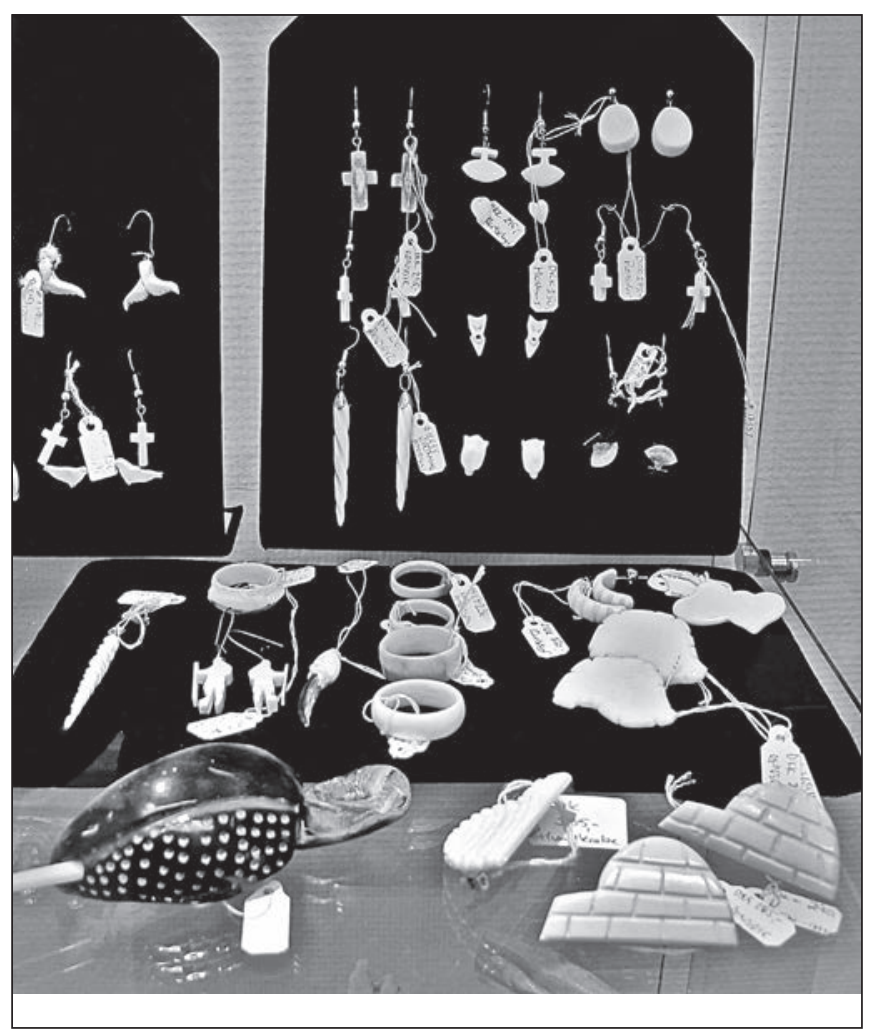

ILLUSTRATION 3 : Objets et bijoux artisanaux d'un magasin de souvenirs, destinés à la vente, dont ceux en forme d'iglou en bois de renne, llulissat (photo : Valérie Kohler).

peau d'estomac d'ours polaire et muni d'une baguette en bois de caribou — remplit trois fonctions : il s'utilise dans le cadre d'une danse où l'on raconte et transmet une histoire; il permet de rendre justice par le rire dans les duels d'affrontement aux tambours; et il constitue un outil pour le chamane. Interdit par les missionnaires au début du XVIII ${ }^{\mathrm{e}}$ siècle lors de la colonisation danoise, le tambour est réintroduit dans les années 1970 au cours du processus de décolonisation progressive. Accompagné de son enfant, un Inuk vivant de la chasse accepte de faire une démonstration de tambour, et de la danse qui l'accompagne, pour des touristes. Attroupés autour de ce chasseur habillé en vêtement d'hiver pour l'occasion, ceux-ci vivent la rencontre de l'Autre comme une expérience concrète (voir illustration 4). "Le tourisme apparaît alors comme le franchissement d'un horizon d'altérité» (Lazzarotti dans Équipe MIT, 2002 : 275). Cette performance a eu lieu sur le bateau de croisière, et non dans le village de ce chasseur visité auparavant.

Dans la perspective de bénéfices socio-économiques, le tourisme arctique traverse une phase transitoire de démarchandisation par la mise en œuvre des principes du développement durable. Cette démarche reste encore opaque pour les voyageurs. Dépréciant l'Autre, le stéréotype et sa justification, alimentés par l'effet de groupe, agissent comme un acte de légitimation d'une situation de domination inconsciemment intégrée par les touristes. Partagée collectivement, l'expérience du voyage peut favoriser le maintien de comportements stéréotypés. Les voyages organisés participent derechef à dédouaner les touristes d'une quelconque responsabilité individuelle face à la durabilité; cette posture tendrait ainsi à renforcer un imaginaire déjà réfractaire à la rapidité des changements.

Les consommateurs de ces espaces accumulent du «... capital culturel, nécessaire à l'affirmation individuelle et à la reconnaissance sociale» (Grenier, 2009 : 9). Le stéréotype n'a pourtant pas qu'un versant négatif; bien qu'il soit potentiellement discriminatoire, il a une fonction régulatrice pour le touriste dans son rapport à l'altérité. Son processus, le stéréotypage ou stéréotypisation, dans la catégorisation et la schématisation de la pensée (Amossy et Herschberg Pierrot, 2011 : 51), est «aussi bien utile que nuisible en fonction des conditions de son usage» (Leyens et al., 1996:30).

N'étant pas le but de leur voyage, la volonté affichée des touristes de s'engager pour «sauver la planète» n'est pas si évidente; elle apparaît le plus souvent sous la forme finale d'une constatation. L'extrait suivant témoigne des sentiments les plus courants des voyageurs pour le Grand Nord :

Pour moi, le Grand Nord, en premier, c'est les grands espaces, c'est la blancheur, le côté désertique, le froid... mais ce n'est pas une question, pour moi, qui évoque la notion climatique... un peu peut-être, mais c'est relativement en arrière-plan, oui... Pour moi, le Grand Nord, c'est les espaces, c'est ce qui s'y passe, c'est aussi tout le côté exploration de ceux qui sont allés chercher le Pôle Nord... (Paul, 63 ans).

Le Grand Nord, c'est d'abord la magie de ces grandes étendues où se sont perdus les premiers explorateurs... voyager là-bas, ça doit être une formidable plongée dans

l'histoire... (Florence, 64 ans).

Si les touristes ne veulent pas voir le moindre signe d'une dégradation dans une zone géographiquement très éloignée de la leur, ils souhaitent plutôt maintenir par tous les moyens l'image d'un Grand Nord dépouillé de tous les signes de leur vie quotidienne. De ce fait, le Grand Nord doit être non seulement vu «avant que ça ne disparaisse» (Paul, 63 ans), mais la protection environnementale rime aussi avec richesse occidentale et modernité, comme si le Grand Nord pouvait s'affranchir de ces principes sous couvert d'une certaine fatalité : «De toute façon, l'écologie et la protection de l'environnement, ce n'est que les pays riches qui peuvent se l'offrir» (Sylvain, 48 ans).

Le discours des touristes semble «se laver» de pratiques paradoxales vis-à-vis d'une écologie pour un tourisme durable; le touriste de nature vient alors assister au spectacle d'un paysage qu'il voudrait sauvegarder grâce à un prétendu écotourisme qui a tendance à s'intensifier. Les écarts entre les principes élaborés par un système de gouvernance et les pratiques et discours touristiques mettent en lumière la subsistance des stéréotypes auprès des voyageurs. Étant donné les objectifs désormais fixés de la durabilité, les exemples cités semblent contredire le prétendu renouvellement du regard porté sur l'Arctique.

Le passage du touriste "contemplatif» au touriste «acteur» construirait l'ébauche d'un acte de réparation au nom d'un tourisme propre qui cherche à se réapproprier le territoire par de bonnes pratiques touristiques. Si le touriste entend le 
discours des problèmes environnementaux actuels d'un point de vue global, le message ne retient pas encore son attention au moment de la restitution spontanée de ses témoignages. Peut-on conclure à l'échec des objectifs du discours de la gouvernance, relayé par l'offre des voyagistes? Les convictions des résidents de l'Arctique pour assurer leur avenir socioéconomique, culturel et environnemental nécessitent l'action d'un tourisme participatif, une solution à mettre en œuvre et en actes pour une conscience accrue de la durabilité. Des signes en faveur de l'environnement sont également pressentis à travers de nouvelles activités : certains voyages sont ainsi proposés pour nettoyer un Arctique pollué - entre autres par les bateaux affrétés ou achetés par les voyagistes — où le touriste deviendrait acteur de et pour la durabilité. Malgré des améliorations locales (Tardif, 2003) possibles, rien ne présage d'un développement économique viable à grande échelle pour ce type de tourisme tourné vers le volontariat. La vraie prise de conscience résiderait-elle alors dans l'initiative des voyagistes? Ne pourraient-ils pas faire visiter des sites anciennement voués aux activités militaires durant la Guerre froide et laissés à l'abandon, comme la DEW Line? Ou encore des anciens sites d'explorations pétrolières, témoins de portions de territoires arctiques dégradés?

L'implication des communautés inuites et la place qu'elles occuperaient dans l'industrie touristique constitueraient aussi une possibilité d'agir en faveur d'un développement durable dans l'Arctique. Le tourisme, intégratif, prendrait ainsi le contre-pied de l'exotisme pour créer une nouvelle dynamique conjointe pour la durabilité.

\section{Conclusion}

Dans le discours des touristes, les changements récents en faveur de la durabilité restent ponctuels, l'idée du Grand Nord étant balisée par des stéréotypes forts. L'Arctique constitue d'abord le fait de vivre l'expérience d'un espace que l'imaginaire collectif a construit depuis longtemps et qu'il faut concrétiser par le voyage. Cette réalisation ne s'effectue pas au risque de perdre la «magie» du Grand Nord qu'ils se représentent, mais participe au contraire à alimenter un imaginaire qu'ils veulent voir perdurer. L'imaginaire ainsi cristallisé fait office de référence dans la valeur d'authenticité attribuée à cet ailleurs-refuge que les touristes veulent se représenter sans contrainte ni obstacle. La durabilité environnementale, comme d'autres questions d'actualité, semble ainsi se dissoudre au profit d'un imaginaire aux fondations solides. Ce dernier n'est néanmoins pas totalement rigide, car il change et évolue de manière malléable au gré de la construction des aspirations et des modes. Influençables et délicates, "[t]he representations of North are as beautiful, powerful, inviting, disturbing, exclusionary, and exploitative as the individuals creating and using them according to accepted standards and ideas of the day» (Grace, $2001: 23$ ).

L'absence de la durabilité dans l'imaginaire collectif trahit donc une certaine imperméabilité du voyageur face aux principes de durabilité présents dans l'offre des voyagistes. Les décalages entre directives de gouvernance et pratiques touristiques, réglementations et comportements lors de visites touristiques, amènent à repenser le rôle tenu par les acteurs du tourisme arctique. Au-delà du constat sur une époque transitoire où se

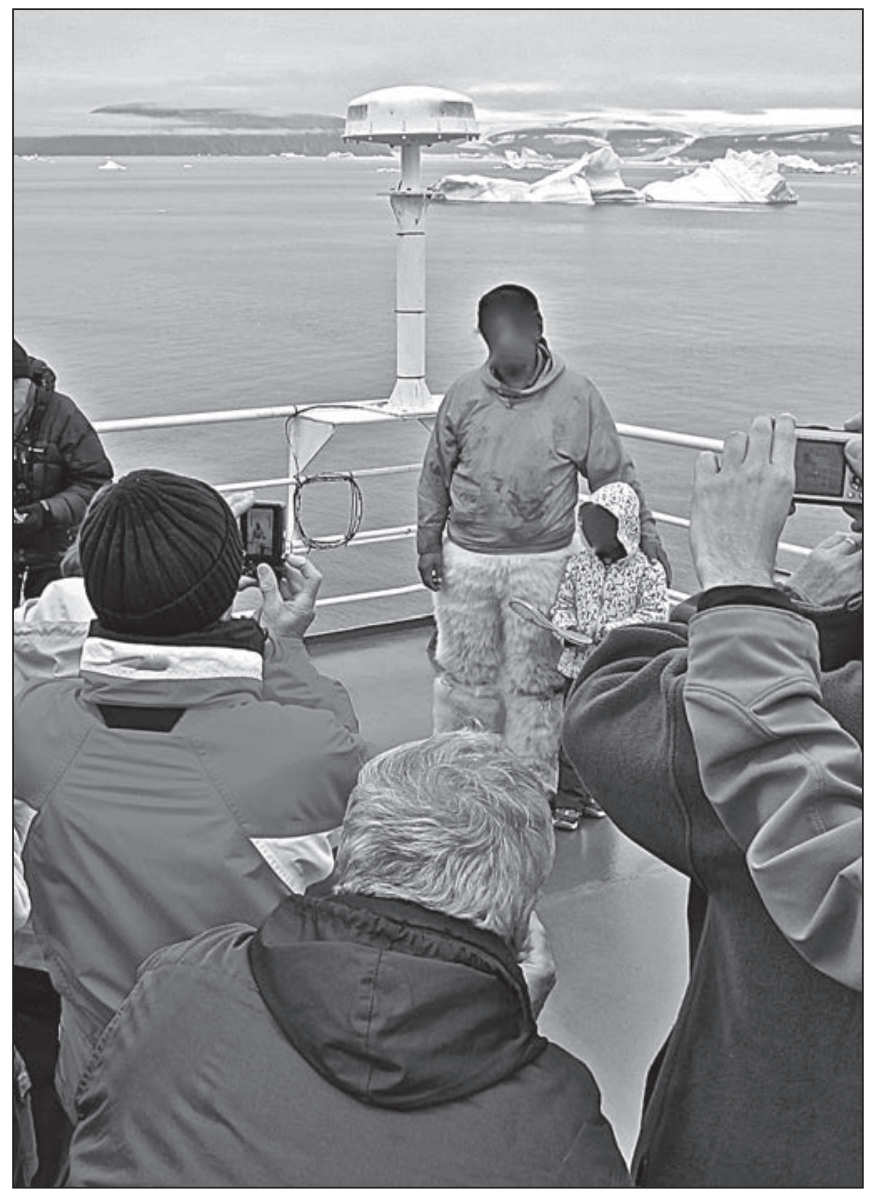

ILLUSTRATION 4 : Vue de touristes photographiant un chasseur groenlandais à Qaanaaq, après sa démonstration de danse au tambour, sur un bateau de croisière touristique (photo : Valérie Kohler).

télescopent les représentations d'un imaginaire postcolonial faisant survivre les stéréotypes avec leur déconstruction par les nouveaux enjeux de la durabilité, c'est la manière de traduire la pensée en actes qui permettrait de redéfinir les défis du tourisme à l'ère de l'après postmodernité.

\section{Références}

AMOSSY, Ruth et Anne HERSCHBERG PIERROT (2011) Stéréotypes et clichés, $3^{\mathrm{e}}$ édition, Paris : Armand Colin. 128 p.

Anløbsliste (2011) Destination Arctic Circle Krydstogter 2011, Visit Greenland - The National Tourist Board, <http://cruise.arcticcircle.gl>, consulté le 14 septembre 2011.

BOURDEAU, Philippe et Libéra BERTHELOT (2008) «Tourisme et Décroissance : de la Critique à l'utopie?», DANS FLIPO. F. et F. Schneider (éd.), Actes de la Première Conférence Internationale sur la Décroissance Economique pour la Soutenabilité et l'Equité Sociale, Paris : 18 et 19 avril 2008, p. 78-86, <http://events.it-sudparis.eu/degrowthconference/appel/>, consulté le $1^{\text {er }}$ juin 2011.

CASTORIADIS, Cornelius (1975) L'Institution imaginaire de la société, Paris : Seuil. 497 p.

CHARTIER, Daniel (2008) Les Nords imaginaires, Montréal : Presses Universitaires du Québec. 340 p. 
COHEN, Erik (1984) «The Sociology of Tourism: Approaches, Issues, and Findings», Annual Review of Sociology, vol. 10, p. 373-392.

COLLIGNON, Béatrice (2005) «Mutations socio-spatiales dans l'Arctique central canadien ", DANS ANDRE, Marie-Françoise (sous la direction de), Le monde polaire. Mutations et transitions, p. 153-165. Paris : Ellipses, Coll. «Carrefours».

DEBARBIEUX, Bernard (1995) «Le lieu, le territoire et trois figures de rhétorique», L’Espace Géographique, vol. 24, n² 2, p. 97-112.

DUPRÉ, Sophie (2009) «Les croisières touristiques dans l'Arctique canadien : Une réalité tangible à l'appropriation territoriale encore limitée», Téoros, vol. $28, \mathrm{n}^{\circ} 1, \mathrm{p} .39-51$.

Équipe MIT (2002) Tourisme 2. Moments de lieux, Paris : Belin, Coll. «Mappemonde». 349 p.

Florida-Caribbean Cruise Association (FCCA) (2011) «Cruise Industry Overview 2011: state of the Cruise Industry», FCCA, $<$ http://www.fcca.com/downloads/2011-overview-book_Cruise\%20Industry\%20 Overview\%20and\%20Statistics.pdf>, consulté le 11 novembre 2011.

FENNELL, David (2008) Ecotourism, $3^{\mathrm{e}}$ édition, New York : Routledge. 302 p. FILLION, Fern, L.; James P. FOLEY et André J. JACQUEMOT (1992) «The Economics of Global Ecotourism ", papier présenté au Quatrième Congrès Mondial sur les Parcs nationaux et les Zones protégées (Caracas, Venezuela : 10 au 21 février), DANS MUNASINGHE, M. et J. MCNEELY (éd.) (1994) Protected area economics and policy: linking conservation and sustainable development, p. 235-252. The World Conservation Bank pour l'Union internationale pour la Conservation de la Nature (UICN).

GÖSSLING, Stefan (2000) «Sustainable Tourism Development in Developing Countries: Some Aspects of Energy Use», Journal of Sustainable Tourism, vol. $8, \mathrm{n}^{\circ} 5$, p. 410-425.

GRABURN, Nelson (2004) «The Invention of Authentic Inuit Art», DANS GRABURN, Nelson et Aaron GLASS (sous la direction de), Beyond Art/Artifact/Tourist Art: Social Agency and the Cultural Value(s) of the Aestheticized Object, Material Culture, vol. 9, n 2, p.141-160.

GRACE, Sherrill (2001) Canada and the Idea of North, Montréal : McGillQueen's Press. $341 \mathrm{p}$.

Grand Nord Grand Large (s. d.) «Grand Nord Grand Large, Le voyage polaire : Notre engagement dans le développement durable», Terres d'Aventure, <http://www.gngl.com/voyage/developpement-durableengagements.aspx $>$, consulté le 8 octobre 2011.

Grands Espaces (s. d.) «À qui s'adressent nos voyages?»<http://www.grandsespaces.ch/croisieres-voyages-destination.php>, consulté le 25 juin 2011.

GRENIER, Alain A. (2008) «Le tourisme de croisière», Téoros, vol. 27, nº 2, p. 36-48.

GRENIER, Alain A. (2009) «Conceptualisation du tourisme polaire : Cartographier une expérience aux confins de l’imaginaire», Téoros, vol. $28, n^{\circ}$ 1, p. 7-19.

HILL, Jennifer et Tim GALE (2009) Ecotourism and Environmental Sustainability: Principles and Practice, Surrey (UK) : Ashgate Publishing Limited. $240 \mathrm{p}$.

JOHNSON, David (2002) «Environmentally sustainable cruise tourism: a reality check», Marine Policy, vol. 26, nº 4, p. 261-270.

KOHLER, Valérie (2011) notes d'observation du 3 août.

KUSLER, Jon A. (1991) «Ecotourism and resource conservation: introduction to issues", DANS KUSLER, Jon A. (sous la direction de), Ecotourism and Resource Conservation: A Collection of Papers, vol. 1, p. 371-381. Madison: Omnipress.

LASSERRE, Frederic (dir.) (2010) Passages et mers arctiques. Géopolitique d'une région en mutation, Québec : Presses de l'Université du Québec, Coll. «Géographie contemporaine». 492 p.
LAZZAROTTI, Olivier (2002) DANS Équipe MIT (2002) Tourismes 1, lieux communs, Paris : Belin, Coll. «Mappemonde». 320 p.

LEYENS, Jacques-Philippe; Vincent YZERBYT et Georges SCHADRON (1996) Stéréotypes et cognition sociale, Wavre : Mardaga,

Coll. «Psychologie et sciences humaines». $310 \mathrm{p}$.

LÜCK, Michael; Patrick T. MAHER et Emma J. STEWART (2010) Cruise tourism in polar regions - Promoting environmental and social sustainability?, Washington : Earthscan. 272 p.

MCGHEE, Robert (2004) The Last Imaginary Place: a Human History of The Arctic World, Toronto : Key Porter Books Limited. 296 p.

MILES, Rick et Karen MILES (cap.) (c2007-2012.) «Home - Eco-tourism», Wanderbird Expedition Cruises, <http://www.wanderbirdcruises.com>, consulté le 11 novembre 2011.

Ministère Danois des Affaires Étrangères (MDAE); Département des Affaires Étrangères du Groenland (DAEG) et Ministère des Affaires Étrangères des Îles Féroé (MAEIF) (2011) Rapport «Kingdom of Denmark Strategy for the Arctic 2011-2020», Copenhague, Nuuk et Tórshavn : Gouvernement du Danemark, du Groenland et des Îles Féroé. 59 p.

MILLER, Kenton R. (1978) Planning National Parks for Ecodevelopment: Methods and Cases from Latin America, vol. 1 et 2, University of Michigan : School of Natural Resources. Center for Strategic Wildland Management Studies. 680 p.

Organisation Mondiale du Tourisme (OMT) (2003) L'activité des croisières dans le monde, Madrid : Éditions OMT. 220 p.

Organisation Mondiale du Tourisme (OMT) (2011) Faits saillants OMT du tourisme, édition 2011, Madrid : Éditions OMT. 12 p.

Port of Longyearbyen Spitsbergen (2012) «Cruise Ship List», Port of Longyearbyen, <http://www.portlongyear.no>, consulté le 12 août 2012.

REMY, Jean (1994) «L'implication paradoxale dans l'expérience touristique», Recherches sociologiques, vol. 25, n², p. 61-78.

SCHAPIRA, Charlotte (1999) Les stéréotypes en français: proverbes et autres formules, Paris : Ophrys. 172 p.SMITH, Melanie K. (2009) Issues in Cultural Tourism Studies, $2^{\mathrm{e}}$ édition, Abingdon : Routledge. 251 p.

STASZAK, Jean-François (2008) «Danse exotique, danse érotique. Perspectives géographiques sur la mise en scène du corps de l'Autre», Les Annales de Géographie, nº66-661, p. 129-158.

Statistics Greenland (1989) «Tourism», Statistics Greenland, <http://www. stat.gl>, consulté le 10 septembre 2011.

TARDIF, Jonathan (2003) «Ecotourisme et développement durable», VertigO, vol. $4, \mathrm{n}^{\circ} 1,<\mathrm{http}: / /$ vertigo.revues.org/index4575.html >, consulté le 12 octobre 2011.

The International Ecotourism Society (TIES) (1990) «Uniting Conservation, Communities and Sustainable Travel», TIES, <www.ecotourism.org>, consulté le 8 septembre 2011.

United Nations (1987) Report of the World Commission on Environment and Development, General Assembly Resolution 42/187, 11 décembre.

URBAIN, Jean-Didier (1993) L'idiot du voyage - Histoires de touristes, Paris : Payot \& Rivages. 270 p.

WANG, Ning (1999) « Rethinking authenticity in tourism experience», Annals of Tourism Research, vol. 26, n 2, p. 349-370.

WEAVER, David B. (2002) «The evolving concept of ecotourism and its potential impacts", International Journal of Sustainable Development, vol. 5, n 3, p. 251-264.

World Expeditions (s. d.) «Adventure holidays on the paths less travelled», World Expeditions - voyagiste australien, <http://www.worldexpeditions.com/au/>, consulté le 11 décembre 2011. 